\title{
Equitable Access to Stroke Care in Canada - The Geographic Conundrum
}

Keywords: Stroke care, Endovascular thrombectomy, Stroke rehabilitation, Stroke access stroke prevention, Stroke thrombolysis, Stroke transport, Stroke geography, Rural stroke, Stroke centers

doi:10.1017/cjn.2020.34

Can J Neurol Sci. 2020; 47: 285-286

Healthcare systems are shaped to best serve the subject population, and the geospatial distribution of the population has an impact on healthcare delivery. The Canadian perspective on stroke care access differs from most of the developed world due to a vast geographic area with low population density. Only four people live per square kilometer of Canada compared to 36 in the USA and 237 in Germany. ${ }^{1}$ Although more than $80 \%$ of Canadians now live in metropolitan or urban centers, rural is still the way of life for every fifth Canadian. Furthermore, $66 \%$ of citizens live within $100 \mathrm{~km}$ from the Canada-US border with $33 \%$ scattered over $96 \%$ of colossal Canada. ${ }^{2}$ The relative concentration of population hubs near the southern border may be good for stroke care access to the majority but makes the other $33 \%$ even more vulnerable. Despite a gradual trend toward urbanization resulting in an increased share of the non-rural population overtime in relative terms, the absolute number of people living rurally is still increasing. ${ }^{3}$

Stroke care must be appropriate, safe, efficient, acceptable, accessible, and effective. ${ }^{4}$ These quality benchmarks are easier to achieve in urban centers, but severe inequalities may appear in rural communities. Lack of access to preventative, restorative, and rehabilitative services in rural Canada can render care non-equitable. Delayed delivery of diagnostic and therapeutic stroke interventions in this time-sensitive disease negatively impacts patient outcomes resulting in care which is neither efficient nor very effective.

In this issue of the Canadian Journal of Neurological Sciences, Kapral et al. explored access to stroke care in rural Ontario communities. ${ }^{5}$ They defined rural as a community with less than 10,000 inhabitants and calculated the total rural population in Ontario as approximately 1.5 million strong. They estimated stroke care access by transport times analyzed for within 30,60, and $240 \mathrm{~min}$ of driving time at posted speeds. Access to stroke care services, including neuroimaging, thrombolysis, endovascular thrombectomy (EVT), stroke unit care, stroke prevention clinics, and inpatient rehabilitation facilities, was assessed.

Their data showed that over $90 \%$ of rural residents lived within $240 \mathrm{~min}$ of drive time for most stroke care services but EVT. They found that EVT access drops rapidly from $83.9 \%$ at $240 \mathrm{~min}$ to $32.1 \%$ at $60 \mathrm{~min}$ and only $3.3 \%$ at less than $30 \mathrm{~min}$ drive. The authors conclude that access to stroke care across rural Ontario communities is reassuringly good with exception of EVT. However, the authors also found a striking access discrepancy between rural residents of northern and southern Ontario across multiple domains of stroke care. For example, almost a third of northern Ontario residents were more than $4 \mathrm{~h}$ from MRI, stroke unit care, and inpatient rehabilitation. Worse still, greater than two thirds were more than $4 \mathrm{~h}$ from EVT. In comparison, no southern Ontario rural residents lived so far from any stroke services. Overall their work is novel in the sense that rural stroke care access has not been previously investigated exclusively.

The data are still somewhat heartening for rural Ontario as a whole, but the picture may not be as rosy across the country. Although $19 \%$ of Canadians lived in a rural area in 2011, the Atlantic provinces and the territories had $40-50 \%$ of their population living rurally. Rural populations in Saskatchewan and Manitoba were also well above the national average. In fact, Ontario and British Columbia are the most urbanized provinces with a rural share of $14 \%$ each. ${ }^{6}$ Stroke care services access will likely fall short of the Ontario average in multiple domains if a similar analysis was conducted for provinces and territories with higher rural proportions and a less organized stroke system.

Although EVT is only one of many stroke care services analyzed in the paper by Kapral et al., it deserves a special mention here. It is an effective restorative intervention for acute large vessel occlusion with the number needed to treat to produce a good outcome as low as $2-3 .^{7}$ EVT access has implications on systems of stroke care and patient outcomes. Expansion of the therapeutic window for EVT from 6 to $24 \mathrm{~h}^{8}$ has made it an uphill task to timely acquire multimodal neuroimaging (including CT angiography and CT perfusion) for patient selection and has intensified the debate surrounding the best way to triage acute stroke transport between primary and comprehensive stroke centers (CSCs). From a rural perspective, determination of EVT eligibility adds the expectation of improved access to these imaging modalities at primary stroke centers with round the clock radiology and tele-stroke network support. Downstream, EVT necessitates stroke observation unit or intensive care unit admission and significantly alters patient care needs. The number of EVTs being performed is on the rise since the publication of thrombectomy trials in 2015 and in the USA, case volumes nearly doubled. ${ }^{9}$ This means CSCs are likely to increase their share in acute phase management. Remote and newly minted CSCs, such as those in northern Ontario, will require adequate funding by the health system and adequate staffing by qualified and available neurointerventionalists as they attempt to provide their populations access to this critical service. Creativity will be required to come up with a physician model that will support these low volume centers while maintaining practitioner competence and site efficiency. Unfortunately, endovascular treatment services are not even available in Yukon Territory, Northwest Territories, or 
Nunavut. ${ }^{10}$ Many other aspects of organized stroke care may also be lacking in these places in which more than 100,000 Canadians abide permanently, many in rural areas. ${ }^{11}$

The results of the current paper suggest that $77 \%$ of rural Ontarians have access to inpatient stroke rehabilitation services within $60 \mathrm{~min}$ of drive time. However, owing to capacity constraints many patients may not qualify for such inpatient care and are rather called for therapy sessions on various schedules. An hour's drive to and from a rehabilitation facility many times each week can result in prohibitive exhaustion for the caregivers and patients. Innovative strategies including early supportive discharge programs and investment in tele-rehabilitation services could bridge such gaps. ${ }^{12}$

Although heavily urbanized, Ontario is home to $25 \%$ of the total rural Canadian populace. The current study not only clearly depicts the strengths of the system of stroke care in Ontario but also highlights opportunities to further improve access. Provincial systems of stroke care must continue to evolve to break down not just urban and rural discrepancies in access to care but discrepancies within rural areas themselves.

\section{Disclosures}

The authors have indicated they have no relevant conflicts of interest to disclose.

\section{Statement of Authorship}

All authors contributed equally to this manuscript.

Asif Butt

Fellow Cerebrovascular Diseases/Stroke, University of Alberta, Edmonton, Canada

Thomas Jeerakathil Division of Neurology, Department of Medicine, University of Alberta, Edmonton, Canada
Correspondence to: Thomas Jeerakathil, Neurology/Medicine, University of Alberta, 7-112G; 11350 - 83rd Avenue, Edmonton, AB T6G 2G3, Canada. Email: thomasj@ualberta.ca

\section{REFERENCES}

1. The World Bank. Population density (people per sq. km of land area). Available at: https://data.worldbank.org/indicator/EN.POP. DNST; accessed Jan 20, 2020.

2. Statistics Canada. Population Size and Growth in Canada: Key Results From the 2016 Census; 2017. Available at: https:// www150.statcan.gc.ca/n1/daily-quotidien/170208/dq170208a-eng. htm; accessed Jan 20, 2020.

3. Macrotrends.net. Canada Rural Population 1960-2020. Available at: https://www.macrotrends.net/countries/CAN/canada/ruralpopulation; accessed Jan 31, 2020.

4. The Alberta Quality Matrix for Health. Available at: https://hqca. ca/about/how-we-work/the-alberta-quality-matrix-for-health-1/; accessed February 2, 2020.

5. Kapral M, Hall R, Gozdyra P, et al. Geographic access to stroke care services in rural communities in Ontario, Canada. Can J Neurol Sci. 2020; 1-18. doi: 10.1017/cjn.2020.9

6. Statistics Canada. Canada goes urban; 2018. Available at: https:// www150.statcan.gc.ca/n1/pub/11-630-x/11-630-x2015004-eng.htm; accessed Jan 31, 2020.

7. Goyal M, Menon BK, van Zwam WH, et al. Endovascular thrombectomy after large-vessel ischaemic stroke: a meta-analysis of individual patient data from five randomised trials. The Lancet. 2016;387(10029):1723-31.

8. Boulanger J, Lindsay M, Gubitz G, et al. Canadian stroke best practice recommendations for acute stroke management: prehospital, emergency department, and acute inpatient stroke care, 6th edition, Update 2018. Int J Stroke. 2018;13(9):949-84.

9. Smith EE, Saver JL, Cox M, et al. Increase in endovascular therapy in get with the guidelines-stroke after the publication of pivotal trials. Circulation. 2017;136(24):2303-10.

10. Eswaradass PV, Swartz RH, Rosen J, et al. Access to hyperacute stroke services across Canadian provinces: a geospatial analysis. CMAJ Open. 2017;5(2):E454-e9.

11. Statistics Canada. Population and dwelling count highlight tables, 2016 census; 2019. Available at: https://www12.statcan.gc.ca/ census-recensement/2016/dp-pd/hlt-fst/pd-pl/Table.cfm? Lang=Eng $\& \mathrm{~T}=101 \& \mathrm{~S}=50 \& \mathrm{O}=\mathrm{A}$; accessed January 31, 2020.

12. Langhorne P, Baylan S. Early supported discharge services for people with acute stroke. Cochrane Database Syst Rev. 2017;7:CD000443. doi: 10.1002/14651858.CD000443.pub4 